\title{
A NON-LOCAL AREA PRESERVING CURVE FLOW
}

\author{
LIANG CHENG AND LI MA*
}

\begin{abstract}
In this paper, we consider a kind of area preserving non-local flow for convex curves in the plane. We show that the flow exists globally, the length of evolving curve is non-increasing, and the curve converges to a circle in $C^{\infty}$ sense as time goes into infinity.
\end{abstract}

\section{INTRODUCTION}

It is a interesting problem to study non-local flow for curves in the plane. The purpose of this paper is to introduce a new non-local flow which preserves the area enclosed by the evolving curve. Our research is motivated by the famous works of Gage and Hamilton [9] and [4] (see also [3] for background and more results). The curve shortening flow in a Riemannian manifold has been studied extensively in the last few decades (see [16]). The curve shortening flow in the plane is the family of evolving curves $\gamma(t)$ such that

$$
\frac{\partial}{\partial t} \gamma(t)=k N
$$

where $k$ and $N$ are the curvature of curve $\gamma$ and the (inward pointing) unit normal vector to the curve. For this flow, deep results are obtained in [9], 7] and [12]. They have proved that a simple closed initial curve remains so along the flow, and the evolving curve becomes more and more circular during the curve shortening process, and it converges to a point in a finite time. Then another natural question arises for expanding evolution flow for curves. B.Chow and D.H. Tsai have studied the expanding flow such as

$$
\frac{\partial}{\partial t} \gamma(t)=-G\left(\frac{1}{k}\right) N
$$

where $G$ is a positive smooth function with $G^{\prime}>0$ everywhere. B.Andrews [1] has studied more general expanding flows, especially flows with anisotropic speeds. They have obtained deep results too. People then like to study curve flow problems preserving some geometric quantities. M.Gage [8] has considered an area-preserving flow

$$
\frac{\partial}{\partial t} \gamma(t)=\left(k-\frac{2 \pi}{L}\right) N
$$

where $L$ is the length of the curve $\gamma$, and have proved that the length of the curve is non-increasing and finally converges to a circle. Based on this, it is interesting study a non-local curve flow which preserves the length of the evolving curve. For this, one may see [17] for a recent study. In a very recent paper [23], S.L.Pan and

1991 Mathematics Subject Classification. 35K15, 35K55, 53A04.

Key words and phrases. non-local flow, area preserving, convex curves, isoperimetric defect.

$*=$ corresponding author. The research is partially supported by the National Natural Science Foundation of China No.11271111 and SRFDP 20090002110019. 
J.N.Yang consider a very interesting length preserving curve flow for convex curves in the plane of the form

$$
\frac{\partial}{\partial t} \gamma(t)=\left(\frac{L}{2 \pi}-k^{-1}\right) N
$$

where $L, N$, and $k$ are the length, unit normal vector,and the curvature of the curve $\gamma(t)$ respectively. They have proved that the convex plane curve will become more and more circular and converges to circle in the $C^{\infty}$ sense.

We now consider the following non-local area preserving curve flow

$$
\frac{\partial}{\partial t} \gamma(t)=\left(\alpha(t)-\frac{1}{k}\right) N,
$$

where $\alpha(t)=\frac{1}{L} \int_{0}^{L} \frac{1}{k} d s$, and obtain the following result.

Theorem 1. Suppose $\gamma(u, 0)$ is a strictly convex curve (i.e. $k(0)>0$ ) in the plane $\mathbb{R}^{2}$. Assume $\gamma(t):=\gamma(u, t)$ satisfies the following evolving equation

$$
\frac{\partial}{\partial t} \gamma(t)=\left(\alpha(t)-\frac{1}{k}\right) N
$$

where $k$ is the curvature of the curve $\gamma(t), N$ is inward pointing unit normal vector to the curve and $\alpha(t):=\alpha(\gamma(t))=\frac{1}{L} \int_{0}^{L} \frac{1}{k} d s$. Then the curve flow problem (1.1) has the global solution $\gamma(t)$, for all $t \in[0, \infty)$. Furthermore, the non-local curve flow(1.1) preserves the area enclosed by the evolving curve and keeps the strictly convexity under the evolution process. More over, $\gamma(t)$ converges to a circle in the $C^{\infty}$ sense as time $t$ goes into infinity.

Note that circles are stationary solutions to (1.1). We may assume that $\gamma(u, 0)$ is not a circle. Otherwise, the result is obvious. The interesting part in the study the non-local flow (1.1) lies in treating the possible collapsing point where $k=\infty$ of the evolving curve at any finite time. To overcome this, we use the maximum principle argument. The behavior of the curve flow is by using the isoperimetric defect property for closed convex curves. For the local existence of the flow, we can decompose the curvature radius function into two parts, which give a linear PDE and nonlinear ODE. We get the local existence of the curve flow by solving the PDE first and then solving the ODE. As a comparison, we shall present the support function trick (15] 22]), which has also been used by B.Chow and B.Andrews in the Gauss curvature flow and in the curve shortening flow. We can show that the global existence of the support functions is equivalent to the globally existence of the non-local flow (1.1). However, this part is new in the research of the non-local flows. The convexity of the evolving flow is proved by the use of maximum principle to the curvature evolution equation. We can show that the curvature of the evolving curve is also uniformly bounded from below by a positive constant. Hence, using the area of the region enclosed by the evolving convex curve is uniformly bounded, we know that the convex region is uniformly contained in a fixed ball. By using Bonnesen inequality (in principle, we may also use John's ellipsoid lemma) [19] 18] we know that the curvature of evolving flow is uniformly bounded and the evolving curve becomes more round, and then we get the global flow. We shall give full proof of this fact in section 3. To prove the convergence of the global flow, we need the argument of Gage-Hamilton [9] (see also the works [6] [7] [10] of Gage and gage and $\mathrm{Yi} \mathrm{Li}$ ). It is not clear to us how to get global existence of the area-preserving non-local flow for curves in non-flat surfaces. 
The paper is organized as follows. In section 2 we calculate some evolution equations related to this curve flow. In section 3, we prove a long time existence for the curve flow (1.1) and show the strictly convexity of the flow is preserved. The local existence of the curve flow is done by using the supporting function method. In section 4 , we show that isoperimetric deficit decays to zero under the non-local curve flow (1.1) and the evolving curve converges to a circle in $C^{\infty}$ sense.

\section{Preparation}

In this section, we calculate some formulae for more general non-local flows than the non-local flow (1.1). Consider the evolving curve $\gamma(t)$ defined by the map $\gamma(u, t): S^{1} \times I \rightarrow \mathbb{R}^{2}$ satisfying the equation:

$$
\frac{\partial}{\partial t} \gamma(t)=\left(\alpha(t)-\frac{1}{k}\right) N
$$

where $\alpha(t)$ is a $C^{\infty}$ function only depends on the time $t$. Since $u$ and $t$ are independent variables, $\frac{\partial}{\partial u}$ and $\frac{\partial}{\partial t}$ commute when applied to functions on $\mathbb{R}^{2}$. Let $s$ denote the arc-length of the curve $\gamma$. Then the operator $\frac{\partial}{\partial s}$ is given in terms of $u$ by

$$
\frac{\partial}{\partial s}=\frac{1}{v} \frac{\partial}{\partial u}
$$

where $v=\left|\frac{\partial \gamma}{\partial u}\right|$.

The arc-length parameter is $d s=v d u$. Let $T$ and $N$ be the unit tangent vector and the (inward pointing) unit normal vectors to the curve respectively. Then the Frenet equations can be written as

$$
\frac{\partial T}{\partial u}=v k N, \quad \frac{\partial N}{\partial u}=-v k T .
$$

We now introduce some formulas according to (2.1). First we have the following evolution equation for $v$.

Lemma 2. Along the flow, it holds that $\frac{\partial v}{\partial t}=(1-k \alpha) v$.

Proof.

$$
\begin{aligned}
\frac{\partial}{\partial t}\left(v^{2}\right) & =\frac{\partial}{\partial t}<\frac{\partial \gamma}{\partial u}, \frac{\partial \gamma}{\partial u}>=2<\frac{\partial \gamma}{\partial u}, \frac{\partial^{2} \gamma}{\partial t \partial u}>=2<\frac{\partial \gamma}{\partial u}, \frac{\partial^{2} \gamma}{\partial u \partial t}> \\
& =2<v T, \frac{\partial}{\partial u}\left(\left(\alpha-\frac{1}{k}\right) N\right)>=2(1-k \alpha) v^{2} .
\end{aligned}
$$

Then the lemma follows immediately.

We also have the following useful relation for the operators $\frac{\partial}{\partial t}$ and $\frac{\partial}{\partial t}$.

Lemma 3. Along the flow, it holds that

$$
\frac{\partial}{\partial t} \frac{\partial}{\partial s}-\frac{\partial}{\partial s} \frac{\partial}{\partial t}=(k \alpha-1) \frac{\partial}{\partial s} \text {. }
$$

Proof.

$$
\begin{aligned}
\frac{\partial}{\partial t} \frac{\partial}{\partial s} & =\frac{\partial}{\partial t}\left(\frac{1}{v} \frac{\partial}{\partial u}\right)=-\frac{v_{t}}{v^{2}} \frac{\partial}{\partial u}+\frac{1}{v} \frac{\partial}{\partial t} \frac{\partial}{\partial u} \\
& =-\frac{v_{t}}{v}\left(\frac{1}{v} \frac{\partial}{\partial u}\right)+\frac{1}{v} \frac{\partial}{\partial u} \frac{\partial}{\partial t}=(k \alpha-1) \frac{\partial}{\partial s}+\frac{\partial}{\partial s} \frac{\partial}{\partial t} .
\end{aligned}
$$


The derivatives of $T$ and $N$ are given by the following result.

Lemma 4. Along the flow, it holds that

$$
\frac{\partial}{\partial t} T=\frac{k_{s}}{k^{2}} N, \quad \text { and } \quad \frac{\partial}{\partial t} N=-\frac{k_{s}}{k^{2}} T .
$$

Proof.

$$
\begin{aligned}
\frac{\partial}{\partial t} T & =\frac{\partial}{\partial t} \frac{\partial}{\partial s} \gamma=\frac{\partial}{\partial s} \frac{\partial}{\partial t} \gamma+(k \alpha-1) \frac{\partial}{\partial s} \gamma \\
& =\frac{\partial}{\partial s}\left(\left(\alpha-\frac{1}{k}\right) N\right)+(k \alpha-1) T \\
& =\left(\frac{\partial}{\partial s}\left(\alpha-\frac{1}{k}\right)\right) N+\left(\alpha-\frac{1}{k}\right) \frac{\partial}{\partial s} N+(k \alpha-1) T \\
& =\frac{k_{s}}{k^{2}} N .
\end{aligned}
$$

The second equation follows from

$$
0=\frac{\partial}{\partial t}<T, N>=<\frac{k_{s}}{k^{2}} N, N>+<T, \frac{\partial}{\partial t} N>,
$$

and $\frac{\partial N}{\partial t}$ must be perpendicular to $\mathrm{N}$.

We denote the angle between the tangent and the $\mathrm{X}$-axis by $\theta$. For convex curves we can use the angle $\theta$ of the tangent line as a parameter. We may write the curvature $k=k(\theta)=\frac{d \theta}{d s}$ in terms of this parameter. Then we have

Lemma 5. Along the flow, it holds that

$$
\frac{\partial \theta}{\partial t}=\frac{k_{s}}{k^{2}} .
$$

Proof. Since $T=(\cos \theta, \sin \theta)$, we use the formula in lemma 4 to calculate

$$
\frac{\partial T}{\partial t}=\frac{k_{s}}{k^{2}} N=\frac{k_{s}}{k^{2}}(-\sin \theta, \cos \theta)
$$

Comparing components on both sides we get the conclusion of this lemma.

The curvature for the evolving curve evolves according to

\section{Lemma 6.}

$$
\frac{\partial k}{\partial t}=\frac{1}{k^{2}} \frac{\partial^{2} k}{\partial s^{2}}-\frac{2}{k^{3}}\left(\frac{\partial k}{\partial s}\right)^{2}+(k \alpha-1) k .
$$

Proof. By lemma 3, we have

$$
\begin{aligned}
\frac{\partial k}{\partial t}=\frac{\partial}{\partial t} \frac{\partial \theta}{\partial s} & =\frac{\partial}{\partial s} \frac{\partial \theta}{\partial t}+(k \alpha-1) \frac{\partial \theta}{\partial s} \\
& =\frac{\partial}{\partial s}\left(\frac{k_{s}}{k^{2}}\right)+(k \alpha-1) k \\
& =\frac{1}{k^{2}} \frac{\partial^{2}}{\partial s^{2}} k-\frac{2}{k^{3}}\left(\frac{\partial}{\partial s} k\right)^{2}+(k \alpha-1) k .
\end{aligned}
$$

This completes the proof.

Denote the area enclosed by the evolving curve by $A(t)$. Then we have 
Lemma 7. $A(t)$ satisfies the equation

$$
\frac{d}{d t} A(t)=\int_{0}^{L} \frac{1}{k} d s-\alpha L
$$

Hence, $A(t)$ remains constant provided $\alpha(t)=\frac{1}{L} \int_{0}^{L} \frac{1}{k} d s$.

Proof. Since

$$
-2 A(t)=\int_{0}^{L}<\gamma, N>d s=\int_{0}^{2 \pi}<\gamma, v N>d u
$$

we have

$$
\begin{aligned}
-2 \frac{d}{d t} A(t)= & \int_{0}^{2 \pi}<\gamma_{t}, v N>+<\gamma, v_{t} N>+<\gamma, v N_{t}>d u \\
= & \int_{0}^{2 \pi}<\left(\alpha-\frac{1}{k}\right) N, v N>d u+\int_{0}^{2 \pi}<\gamma,(1-k \alpha) v N>d u \\
& +\int_{0}^{2 \pi}<\gamma,\left(-\frac{k_{s}}{k^{2}}\right) v T>d u \\
= & \int_{0}^{2 \pi}\left(\alpha-\frac{1}{k}\right) v d u+\int_{0}^{2 \pi}<\gamma,(1-k \alpha) v N>d u \\
& +\int_{0}^{2 \pi} \frac{\partial}{\partial u}\left(\frac{1}{k}-\alpha\right)<\gamma, T>d u \\
= & \int_{0}^{L}\left(\alpha-\frac{1}{k}\right) d s+\int_{0}^{2 \pi}<\gamma,(1-k \alpha) v N>d u \\
& +\int_{0}^{2 \pi} \frac{\partial}{\partial u}\left(\frac{1}{k}-\alpha\right)<\gamma, T>d u
\end{aligned}
$$

By the use of integration by parts, we have

$$
\begin{aligned}
-2 \frac{d}{d t} A(t)= & \int_{0}^{L}\left(\alpha-\frac{1}{k}\right) d s+\int_{0}^{2 \pi}<\gamma,(1-k \alpha) v N>d u \\
& +\int_{0}^{2 \pi}\left(\alpha-\frac{1}{k}\right)\left(<\gamma_{u}, T>+<\gamma, T_{u}>\right) d u \\
= & \int_{0}^{L}\left(\alpha-\frac{1}{k}\right) d s+\int_{0}^{2 \pi}<\gamma,(1-k \alpha) v N>d u \\
& +\int_{0}^{2 \pi}\left(\alpha-\frac{1}{k}\right)(<v T, T>+<\gamma, v k N>) d u \\
= & 2 \int_{0}^{L}\left(\alpha-\frac{1}{k}\right) d s=-2\left(\int_{0}^{L} \frac{1}{k} d s-\alpha L\right) .
\end{aligned}
$$

A useful lower bound for $\alpha(t)$ in the flow (1.1) is below.

Lemma 8. If $\alpha(t)=\frac{1}{L} \int_{0}^{L} \frac{1}{k} d s$, we have

$$
\alpha \geq \frac{L}{2 \pi} .
$$

The equality holds if and only if the curve $\gamma$ has the constant curvature. 
Proof. Since

$$
\int_{0}^{L} k d s=2 \pi
$$

using the Cauchy-Schwartz inequality we have

$$
\int_{0}^{L} k d s \cdot \int_{0}^{L} \frac{1}{k} d s \geq\left(\int_{0}^{L} d s\right)^{2}=L^{2} .
$$

Then we have the result.

Lemma 9. The length of the evolving curve evolves by

$$
\frac{d}{d t} L=L-2 \pi \alpha(t)
$$

Moreover, $\frac{d}{d t} L \leq 0$ provided $\alpha(t)=\frac{1}{L} \int_{0}^{L} \frac{1}{k} d s$.

Proof.

$$
\frac{d}{d t} L=\int_{0}^{2 \pi} v_{t} d u=\int_{0}^{2 \pi}(1-k \alpha) v d u=\int_{0}^{L}(1-k \alpha) d s=L-2 \pi \alpha .
$$

We have $\frac{d}{d t} L \leq 0$ if $\alpha(t)=\frac{1}{L} \int_{0}^{L} \frac{1}{k} d s$ by lemma 8

So much for the general flow (2.1).

\section{LOCAL AND LONG TIME EXISTENCE}

We first consider a priori estimates of the curve flow. Since the changing of the tangential components of the velocity vector of $\gamma_{t}$ affects only the parametrization, not the geometric shapes of the evolving curve, we can choose a suitable tangent component $\eta$ to simplify the analysis of the non-local flow (1.1). This trick has been used by many authors, see, for example, 9] or [23. So we consider the following evolution problem, which is equivalent to (1.1):

$$
\gamma_{t}=\left(\alpha(t)-\frac{1}{k}\right) N+\eta T .
$$

Similar to the calculations in section 2 we have

Lemma 10. Along the flow (3.1), it is true that

$$
\begin{aligned}
& \frac{\partial v}{\partial t}=\frac{\partial \eta}{\partial u}+(1-k \alpha) v, \\
& \frac{\partial}{\partial t} T=\left(\eta k+\frac{k_{s}}{k^{2}}\right) N, \quad \frac{\partial}{\partial t} N=-\left(\eta k+\frac{k_{s}}{k^{2}}\right) T, \\
& \frac{\partial \theta}{\partial t}=\eta k+\frac{k_{s}}{k^{2}}, \\
& \frac{\partial k}{\partial t}=\frac{1}{k^{2}} \frac{\partial^{2} k}{\partial s^{2}}-\frac{2}{k^{3}}\left(\frac{\partial k}{\partial s}\right)^{2}+(k \alpha-1) k+\eta \frac{\partial k}{\partial s} \\
& \frac{d}{d t} A(t)=\int_{0}^{L} \frac{1}{k} d s-\alpha L, \\
& \frac{d}{d t} L=L-2 \pi \alpha .
\end{aligned}
$$


Note that $L$ and $A$ are both independent of $\eta$. In order to make $\theta$ independent of time $t$, we can choose suitable $\eta$ such that $\frac{\partial \theta}{\partial t}=0$, i.e.

$$
\eta=-\frac{1}{k^{3}} k_{s}=-\frac{1}{k^{2}} \frac{\partial k}{\partial \theta} \text {. }
$$

Then by changing the space variable we can transform away the tangential component, without changing the shape of the curves (see also the proof of Theorem 4.1.4 in 9]). Hence we can get from the flow (3.1) the flow (1.1).

We now consider the following equivalent problem instead from now on:

$$
\gamma_{t}=\left(\alpha(t)-\frac{1}{k}\right) N-\frac{1}{k^{3}} \frac{\partial k}{\partial s} T .
$$

Then by lemma 10, we have the following result.

Lemma 11. Along the flow (3.2), it holds that

$$
\begin{aligned}
& \frac{\partial}{\partial t} T=0, \quad \frac{\partial}{\partial t} N=0, \quad \frac{\partial \theta}{\partial t}=0, \\
& \frac{\partial k}{\partial t}=\frac{1}{k^{2}} \frac{\partial^{2} k}{\partial s^{2}}-\frac{3}{k^{3}}\left(\frac{\partial k}{\partial s}\right)^{2}+(k \alpha-1) k, \\
& \frac{d}{d t} A(t)=\int_{0}^{L} \frac{1}{k} d s-\alpha L, \\
& \frac{d}{d t} L=L-2 \pi \alpha .
\end{aligned}
$$

By theorem 14, we can use the angle variable $\theta$ of the tangent line as a parameter for convex curves. To determine the evolution equation for curvature of the evolving curve when using $\theta$ as a parameter, we take $\tau=t$ as the time parameter. That is, we change variables from $(u, t)$ to $(\theta, \tau)$. We obtain the following equation for $k$ in terms of $\theta$ and $\tau$.

\section{Lemma 12.}

$$
\frac{\partial k}{\partial \tau}=\frac{\partial^{2} k}{\partial \theta^{2}}-\frac{2}{k}\left(\frac{\partial k}{\partial \theta}\right)^{2}+(k \alpha-1) k .
$$

Proof. By the chain rule and lemma 11, we have

$$
\frac{\partial k}{\partial t}=\frac{\partial k}{\partial \tau}+\frac{\partial k}{\partial \theta} \frac{\partial \theta}{\partial t}=\frac{\partial k}{\partial \tau}
$$

and

$$
\frac{\partial^{2} k}{\partial s^{2}}=\left(\frac{\partial \theta}{\partial s} \frac{\partial}{\partial \theta}\right)\left(\frac{\partial \theta}{\partial s} \frac{\partial}{\partial \theta}\right)=k^{2} \frac{\partial^{2} k}{\partial \theta^{2}}+k\left(\frac{\partial k}{\partial \theta}\right)^{2} .
$$

Substituting these expressions into the formula (3.4) in lemma 11 we get the result.

Note that $L=\int_{S^{1}} \frac{1}{k} d \theta$. By direct calculation, we can derive a heat equation for $1 / k$ (see (3.9) ) from formula (3.7).

Lemma 13. We have

$$
\frac{\partial}{\partial \tau}\left(\frac{1}{k}\right)=\frac{\partial^{2}}{\partial \theta^{2}}\left(\frac{1}{k}\right)+\frac{1}{k}-\alpha .
$$

Let $h=\frac{1}{k}-\frac{L}{2 \pi}$ and let $w=h e^{-\tau}$. Then we have

$$
h_{\tau}=h_{\theta \theta}+h
$$


and

$$
w_{\tau}=w_{\theta \theta} .
$$

Then $w$ can be solved for time interval $[0,+\infty)$ as

$$
w(\theta, \tau)=\int_{-\infty}^{\infty} \frac{1}{2 \sqrt{\pi \tau}} e^{-\frac{(\theta-\xi)^{2}}{4 \tau}} w(\theta, 0) d \xi
$$

and the solution to the flow (1.1) is smooth.

Proof. Since

and

$$
\frac{\partial}{\partial \tau}\left(\frac{1}{k}\right)=-\frac{k_{\tau}}{k^{2}}=-\frac{k_{\theta \theta}}{k^{2}}+\frac{2}{k^{3}} k_{\theta}^{2}-\frac{k^{2} \alpha-k}{k^{2}},
$$

$$
\frac{\partial^{2}}{\partial \theta^{2}}\left(\frac{1}{k}\right)=-\frac{\partial}{\partial \theta}\left(\frac{k_{\theta}}{k^{2}}\right)=-\frac{k_{\theta \theta}}{k^{2}}+\frac{2}{k^{3}} k_{\theta}^{2},
$$

(3.8) follows immediately.

By lemma 9, we have

$$
\begin{aligned}
h_{\tau} & =\frac{\partial}{\partial \tau}\left(\frac{1}{k}\right)-\frac{L_{\tau}}{2 \pi}=\frac{\partial^{2}}{\partial \theta^{2}}\left(\frac{1}{k}\right)+\frac{1}{k}-\alpha-\frac{L-2 \pi \alpha}{2 \pi} \\
& =\frac{\partial^{2}}{\partial \theta^{2}}\left(\frac{1}{k}\right)+\left(\frac{1}{k}-\frac{L}{2 \pi}\right) \\
& =h_{\theta \theta}+h .
\end{aligned}
$$

Then (3.9) follows immediately.

By lemma 13 we know that the function $h$ is globally well-defined from the initial data $h(0)$ of the curve $\gamma(0)$. Note that $\int_{S^{1}} h d \theta=0$. Using $\frac{1}{k}=h+\frac{L}{2 \pi}$ and $\alpha=\frac{1}{L} \int_{0}^{L} \frac{1}{k} d s$, we know that

$$
\alpha=\frac{1}{L} \int_{0}^{2 \pi} \frac{1}{k^{2}} d \theta=\frac{1}{L} \int_{S^{1}} h^{2} d \theta+\frac{L}{2 \pi} .
$$

Then from the ODE

$$
\frac{d}{d \tau} L=L-2 \pi \alpha=-\frac{1}{L} \int_{S^{1}} h^{2} d \theta
$$

we can solve $L$ from the initial curve $\gamma(0)$ and then we get $\frac{1}{k}$ in local time interval. Then, we can get the local existence of the curve flow via the formula

$$
x(\theta, t)=\int_{0}^{\theta} \frac{\cos \phi}{k} d \phi, \quad y(\theta, t)=\int_{0}^{\theta} \frac{\sin \phi}{k} d \phi
$$

to define evolving curves $\gamma(t)$ (as in [9]) for the flow equation (3.2). As a comparison, we shall try to consider the local existence by using the supporting function method since it is often used in the literatures about curve evolution flows.

Theorem 14. Under the assumptions of theorem 1, the curve flow keeps the convexity property.

Proof. By lemma 13 there exists a constant $M>0$ such that for $(\theta, \tau) \in[0,2 \pi] \times$ $(0, \infty)$,

$$
|w(\theta, \tau)| \leq M
$$


Then for any finite $T^{*}, \tau \in\left[0, T^{*}\right)$,

$$
\left|\frac{1}{k}-\frac{L}{2 \pi}\right| \leq M e^{T^{*}}
$$

By lemma 9, L is bounded above. Also by the isoperimetric inequality, L has a lower bound $\sqrt{4 \pi A}$. So we get

$$
k(\theta, \tau) \neq 0, \text { for }(\theta, \tau) \in[0,2 \pi] \times\left[0, T^{*}\right) .
$$

Now, from the continuity of $k(\theta, \tau)$ and the positivity of $k(\theta, 0)$, we know that

$$
k(\theta, \tau)>0, \text { for }(\theta, \tau) \in[0,2 \pi] \times\left[0, T^{*}\right) .
$$

Then the theorem follows from the arbitrariness of $T^{*}$.

One can also see that $\frac{1}{k}$ is uniformly bounded at any finite existing time interval $[0, T)$. This then implies that $\alpha(\tau) \leq C(T)$ for some constant $C(T)>0$. In fact, by the maximum principle we know that $\inf _{S^{1}} w(\theta, \tau)$ is non-decreasing and $\sup _{S^{1}} w(\theta, \tau)$ is non-increasing. This implies that

$$
\frac{1}{k}-\frac{L}{2 \pi} \leq\left[\frac{1}{k(0)}-\frac{L(0)}{2 \pi}\right] e^{\tau}
$$

Then

$$
\frac{1}{k} \leq \frac{L}{2 \pi}+\sup _{S^{1}}\left[\frac{1}{k(0)}-\frac{L(0)}{2 \pi}\right] e^{\tau},
$$

which gives a lower bound of $k$ in any time interval. Here we have used the fact that $\int_{\gamma} k(0) d s=2 \pi$, which gives $L(0) \inf _{S^{1}} k(0)<2 \pi\left(\right.$ and $\sup _{S^{1}} k(0)^{-1}=$ $\left.(\inf k(0))^{-1}>\frac{L(0)}{2 \pi}\right)$ unless $\gamma(0)$ is the circle. Similarly, we have

$$
\frac{1}{k} \geq \frac{L}{2 \pi}+\inf _{S^{1}}\left[\frac{1}{k(0)}-\frac{L(0)}{2 \pi}\right] e^{\tau} .
$$

More importantly, these two estimates imply that there is no blow-up of the evolving curve in any finite time interval.

By this, we have proved the existence of global flow of (2.1).

Theorem 15. Assume the local existence of the curve flow (2.1). We have a global flow to the curve flow (2.1), that is, there is no finite time blow up point of the curvature function $k$.

Now we present the supporting function method to prove the local existence of the curve flow (2.1) (see theorem I1.2 in [1]). We denote $S$ the support function of the curve $\gamma$, i.e. $S=-\left\langle\gamma, N>\right.$. So, $L=\int_{S^{1}} S d \theta$ and

$$
\frac{1}{k}=\frac{\partial^{2} S}{\partial \theta^{2}}+S
$$

Since $\alpha=\frac{1}{L} \int_{0}^{L} \frac{1}{k} d s$, we then have

$$
\alpha=\frac{1}{L} \int_{S^{1}}\left(\partial_{\theta}^{2} S+S\right)^{2} d \theta
$$

We have the following evolution equation of support function.

Lemma 16.

$$
\frac{\partial S}{\partial \tau}=\frac{\partial^{2} S}{\partial \theta^{2}}+S-\alpha
$$


Proof. By lemma 11, we have

$$
\begin{aligned}
\frac{\partial S}{\partial \tau} & =-\frac{\partial}{\partial \tau}<\gamma, N>=-<\frac{\partial}{\partial \tau} \gamma, N> \\
& =-<\left(\alpha(\tau)-\frac{1}{k}\right) N-\frac{1}{k^{2}} \frac{\partial k}{\partial \theta} T, N> \\
& =\frac{1}{k}-\alpha \\
& =\frac{\partial^{2} S}{\partial \theta^{2}}+S-\alpha .
\end{aligned}
$$

Then we have

$$
\left[\left(S-\frac{L}{2 \pi}\right) e^{-\tau}\right]_{\tau}=\left[\left(S-\frac{L}{2 \pi}\right) e^{-\tau}\right]_{\theta \theta} .
$$

Similar to lemma 13, we have

Theorem 17. The support function $S$ can be solved for time interval $[0,+\infty)$ as

$$
\left(S(\theta, \tau)-\frac{L(\tau)}{2 \pi}\right) e^{-\tau}=\int_{-\infty}^{\infty} \frac{1}{2 \sqrt{\pi \tau}} e^{-\frac{(\theta-\xi)^{2}}{4 \tau}}\left(S(\theta, 0)-\frac{L(0)}{2 \pi}\right) d \xi
$$

Furthermore,

$$
\left(\frac{1}{k}-S(\theta, \tau)\right) e^{-\tau}=\int_{-\infty}^{\infty} \frac{1}{2 \sqrt{\pi \tau}} e^{-\frac{(\theta-\xi)^{2}}{4 \tau}}\left(\frac{1}{k}(0)-S(\theta, 0)\right) d \xi .
$$

From all these, we can easily get the following.

Theorem 18. For $\tau>0$, we have

$$
\left(\frac{1}{k}-S\right)(\theta, \tau) \geq e^{\tau} \inf _{\theta}\left(\frac{1}{k}(0)-S(\theta, 0)\right) .
$$

With these understanding, we can use the general existence result of Jiang- Pan 15. (or the method used in [17]) to show that there is a local solution to the flow (3.11). It is convenient to choose the normal vector for parameter of the curve. We denote $\mathbf{n}: \gamma \rightarrow S^{1}$ be the Gauss map. Let $z$ be the normal vector and $\gamma$ parametrized by $z$. So we have $S(z, t)=-<\gamma\left(n^{-1}(z)\right), z>$. Let $r[S](z)$ be the radius of curvature at the point with normal $z$ is given by $r[S](z)=\frac{\partial^{2} S}{\partial \theta^{2}}(z)+S(z)$. Then we obtain the following result.

Theorem 19. Assume $S: S^{1} \times[0, \infty) \rightarrow \mathbb{R}$ is a smooth function of equation (3.11) with radius $r[S]>0$, then there exists a solution $\gamma: \zeta \times[0, \infty) \rightarrow \mathbb{R}$ satisfies the equation (1.1) which has the initial data $\gamma_{0}=\left\{-S_{0}(z) z-\frac{\partial S_{0}}{\partial \theta}(z) \frac{\partial z}{\partial \theta}: z \in S^{1}\right\}$ and such that the curve $\gamma(t)$ has the support function $S(t)$ for each $t \in[0, \infty)$.

Proof. We define the evolving curve $\bar{\gamma}: S^{1} \times[0, \infty) \rightarrow \mathbb{R}$ by

$$
\bar{\gamma}(z, t)=-S(z, t) z-\frac{\partial S}{\partial \theta}(z, t) \frac{\partial z}{\partial \theta} .
$$


Then we have an evolving curve $\gamma(t)$ which has the support function $S$ and the curvature $k$ satisfying (3.10). By the assumptions of this theorem,

$$
\begin{aligned}
\frac{\partial \bar{\gamma}}{\partial t}(z, t) & =-\frac{\partial S}{\partial t}(z, t) z-\frac{\partial^{2} S}{\partial t \partial \theta} \frac{\partial z}{\partial \theta} \\
& =-\left(\frac{\partial^{2} S}{\partial \theta^{2}}+S-\alpha\right) z-\frac{\partial}{\partial \theta}\left(\frac{\partial^{2} S}{\partial \theta^{2}}+S-\alpha\right) \frac{\partial z}{\partial \theta} \\
& =(\alpha-r[S](z)) z-\frac{\partial}{\partial \theta}(r[S](z)) \frac{\partial z}{\partial \theta} \\
& =\left(\alpha-\frac{1}{k_{\bar{\gamma}}}\right) N_{\bar{\gamma}}(z)-T \bar{\gamma}(V),
\end{aligned}
$$

where $N_{\bar{\gamma}}$ and $k_{\bar{\gamma}}$ are the normal and curvature corresponding to $\bar{\gamma}$, and $V \in$ $T S^{1} \times[0, \infty)$ is the vector field on $S^{1}$ given by $k_{\bar{\gamma}} \frac{\partial}{\partial \theta}\left(\frac{1}{k_{\bar{\gamma}}}\right) \frac{\partial z}{\partial \theta}$. Here we used the fact $T \bar{\gamma}(V)=k_{\bar{\gamma}}^{-1} V$ for any $V \in T S^{1}$. Next we define a family of diffeomorphisms $\phi$ such that $\gamma(p, t)=\bar{\gamma}(\phi(p, t), t)$ gives the solution of equation (1.1). Now we take $\phi(p, t)$ to solve the following ordinary differential equation for each $p$ :

$$
\frac{d}{d t} \phi(p, t)=V(\phi(p, t), t)
$$

This equation has a unique solution for each $p$ as long as $S$ exists and remains smooth. Then we have

$$
\begin{aligned}
\frac{\partial}{\partial t} \gamma(p, t) & =\frac{\partial}{\partial t} \bar{\gamma}(\phi(p, t), t) \\
& =\left(\frac{\partial}{\partial t} \bar{\gamma}\right)(\phi(p, t), t)+T \bar{\gamma}\left(\frac{\partial}{\partial t} \phi(p, t), t\right) \\
& =\left(\alpha-\frac{1}{k_{\bar{\gamma}}(\phi(p, t), t)}\right) N_{\bar{\gamma}}(\phi(p, t), t)-T \bar{\gamma}(V)+T \bar{\gamma}(V) \\
& =\left(\alpha-\frac{1}{k_{\gamma}(p, t)}\right) N_{\gamma}(p, t),
\end{aligned}
$$

where we have used $k_{\gamma}(p, t)=k_{\bar{\gamma}}(\phi(p, t), t)$ and $N_{\gamma}(p, t)=N_{\bar{\gamma}}(\phi(p, t), t)$. Hence the theorem holds.

The above result implies the local existence of the curve flow. Hence we get the following result immediately by the use of theorem 17 and theorem 19.

Theorem 20. Under the assumptions of theorem 1, the curve flow (1.1) has the global solution, that the flow exists in time interval $[0, \infty)$ with initial curve $\gamma(0)$.

\section{Convergence}

In this section we prove the convergence of the evolving curves.

In order to understand the behavior of the global curve flow, we need the following isoperimetric inequality due to S.L.Pan and J.N.Yang.

Theorem 21. 23. For the closed, convex $C^{2}$ curves in the plane, we have

$$
\frac{L^{2}-2 \pi A}{\pi} \leq \int_{0}^{L} \frac{1}{k} d s
$$

where $L, A$ and $k$ are the length of the curve, the area enclosed by the evolving curve, and its curvature. 
Recall the following Bonnesen inequality ([19]) that

$$
L^{2}-4 \pi A \geq A^{2}\left(\frac{1}{r_{i n}}-\frac{1}{r_{o u}}\right)^{2},
$$

where $r_{i n}$ and $r_{\text {ou }}$ are radii of the incircle (the largest circle contained in the domain enclosed by $\gamma$ ) and the circumcircle ( the smallest circle containing $\gamma$ ). Since along the flow, the area is fixed and then the curve $\gamma$ becomes more round provided the isoperimetric deficit $L^{2}-4 \pi A$ is non-increasing.

In fact, we have the following result, which shows that the curve flow becomes more and more circular under the evolution process.

Theorem 22. If a convex curve evolves according to (2.1), then the isoperimetric deficit $L^{2}-4 \pi A$ is non-increasing during the evolution process and in case of global flow, it converges to zero as the time $\tau$ goes to infinity.

Proof. By lemma 7 and lemma 9, we have

$$
\begin{aligned}
\frac{d}{d \tau}\left(L^{2}-4 \pi A\right) & =2 L L_{t}-4 \pi A_{\tau} \\
& =2 L(L-2 \pi \alpha)-4 \pi\left(\int_{0}^{L} \frac{1}{k} d s-\alpha L\right) \\
& =2 L^{2}-4 \pi \int_{0}^{L} \frac{1}{k} d s .
\end{aligned}
$$

By the theorem 21, we have

$$
\frac{d}{d t}\left(L^{2}-4 \pi A\right) \leq 2 L^{2}-4 \pi \frac{L^{2}-2 \pi A}{\pi} \leq-2\left(L^{2}-4 \pi A\right),
$$

We always have $L^{2}-4 \pi A \geq 0$, and so

$$
\frac{d}{d \tau}\left(L^{2}-4 \pi A\right) \leq 0
$$

Moreover, we have

$$
0 \leq L^{2}-4 \pi A \leq C \exp (-2 \tau),
$$

where $C=L^{2}(0)-4 \pi A(0)$. As $t \rightarrow \infty$ in case of global flow, we have the decay of the isoperimetric defect,

$$
L^{2}-4 \pi A \rightarrow 0
$$

Recall that the area is fixed along our curve flow. By (4.1), we know that the isoperimetric defect for closed convex curve is the measure of circularness of the curve (see [19] and [20] for more related inequalities). Then we know that for $\tau>0$, $\gamma(\tau)$ is more circular than $\gamma(0)$ but with fixed area of their enclosed regions.

We now give a remark for higher derivative bounds of $k$. We may also get higher order derivatives estimates for $1 / k$. Let $\left(\frac{1}{k}\right)_{\theta}=\partial_{\theta}\left(\frac{1}{k}\right)$. Then we have

or

$$
\frac{\partial}{\partial \tau}\left(\frac{1}{k}\right)_{\theta}=\frac{\partial^{2}}{\partial \theta^{2}}\left(\frac{1}{k}\right)_{\theta}+\left(\frac{1}{k}\right)_{\theta}
$$

$$
\frac{\partial}{\partial \tau}\left[e^{-\tau}\left(\frac{1}{k}\right)_{\theta}\right]=\frac{\partial^{2}}{\partial \theta^{2}}\left[e^{-\tau}\left(\frac{1}{k}\right)_{\theta}\right] .
$$


Using the maximum principle, we know that $\left(\frac{1}{k}\right)_{\theta}$ is bounded at any existing time. In fact we have

$$
\left(\frac{1}{k}\right)_{\theta} e^{-\tau}=\int_{-\infty}^{\infty} \frac{1}{2 \sqrt{\pi \tau}} e^{-\frac{(\theta-\xi)^{2}}{4 \tau}}\left(\frac{1}{k}\right)_{\theta}(0) d \xi
$$

We denote $r_{i n}$ radii of the largest inscribed circle of the curve $\gamma$. Now we can use the method of M.Gage and R.S.Hamilton to show curvature $k$ converging to a constant as time goes into infinity, see Section 5 in 9 . First, we need a result in [9].

Theorem 23. 9] $k(\theta, t) r_{i n}(t)$ converges uniformly to 1 , when the isoperimetric deficit $L^{2}-4 \pi A \rightarrow 0$.

Theorem 24. Under the assumptions of theorem 1, we have $k \rightarrow \frac{2 \pi}{L}$ as $t \rightarrow \infty$.

Proof. By the Bonnesen inequality (see [19]),

$$
\frac{L^{2}}{A}-4 \pi \geq \frac{\left(L-2 \pi r_{i n}\right)^{2}}{A}
$$

and theorem 22, theorem 17, we have $r_{i n} \rightarrow \frac{L}{2 \pi}$ as $t \rightarrow \infty$. Hence the theorem follows immediately from theorem 23 .

Then we obtain the $C^{\infty}$ convergent part in theorem 1 .

Theorem 25. Under the assumptions of theorem 1, the curve flow (1.1) converges to a circle in $C^{\infty}$ sense as time goes into infinity.

Proof. By lemma 13, the curvature $k(t)$ is $C^{\infty}$ differentiable. Then theorem 1 follows immediately from theorem 24 .

\section{REFERENCES}

[1] B.Andrews, Evolving convex curves. Calc.Var.PDE's, 7,315-371(1998).

[2] T.Bonnesen, W.Fenchel, Theorie der Convexen Körper.Chelsea,New York, 1948.

[3] K.S.Chou, X.P.Zhu, The curve shortening Problem. CRC Pre ss.Boca Raton, 2001.

[4] B.Chow, D.H.Tsai, Geometric expansion of convex plane curves. J.Diff.Geom.,44,312330(1996)

[5] B.Chow, P.Lu,L.Ni, Hamilton's Ricci Flow. Science Press/American Mathematical Society, Beijing/Providence,(2006).

[6] M.Gage, An isoperimetric inequality with applications to curve shortening. Duke Math.J.,50,1225-1229(1983).

[7] M.Gage, Curve shortening makes convex curves circular. Invent.Math., 76(1984) 357-364

[8] M.Gage, On an area-preserving evolution equation for plane curves. In:DeTurck,D.M.(ed,) Nonlinear Problems in Geometry,Contemp.Math,vol.51,pp.51-62(1986)

[9] M.Gage, R.S.Hamilton, The heat equation shrinking convex plane curves. J.Diff.Geom., 23,6996(1986).

[10] M.Gage, Yi Li, Evolving plane curves by curvature in relative geometries. II. Duke Math. J. 75 (1994), no. 1, 79-98.

[11] M.Green, S.Osher, Steiner polynomials, Wulff flows, and some new isoperimetric inequalities for convex plane curves. Asian J. Math. 3, 659-676 (1999)

[12] M.Grayson, The heat equation shrinks embeded plane curves to round points. J.Diff.Geom.26,285-314(1987).

[13] G.Huisken, Flow by mean curvature of convex surfaces into spheres. J.Diff.Geom.,20,237266(1984).

[14] G.Huisken, The volume preserving mean curvature flow. J. Reine Angew. Math., 382, 35-48 (1987)

[15] L.S.Jiang, S.L.Pan, On a non-local curve evolution problem in the plane. Commun. Anal. Geom. 16, 1-26 (2008) 
[16] L.Ma, D.Z.Chen, Curve shortening in a Riemannian manifold. Ann.Mat.Pura.Appl., 186,663-684(2007).

[17] L.Ma, A.Q.Zhu, On a length preserving curve flow. Monatsh Math (2012) 165:57-78

[18] De G. Miguel, Differentiation of integrals in Rn; Lectures in Math. 481,(1977, SpringerVerlag.

[19] R.Osserman, Bonnesen isoperimetric inequalities. Amer.Math.Monthly, 86(No.1)(1979)1-29.

[20] R.Schneider, Convex bodies: The Brunn-Minkowski theory. Encyclopedia of Mathematics and its Applications Vol. 44, Cambridge University Press, (1993).

[21] Grisha Perelman, Finite time extinction for the solutions to the Ricci flow on certain threemanifold. math.DG/0307245 2003.

[22] S.L. Pan, A note on the general curve flows. J. Math. Stud. 33, 17-26 (2000)

[23] S.L. Pan, J.N. Yang, On a non-local perimeter-preserving curve evolution problem for convex plane curves. manuscripta math.,127,(2008)469-484.

[24] X.P.Zhu, Lectures on mean curvature flows. AMS/IP Studies in Advanced Mathematics, vol. 32. American Mathematical Society/International Press, Providence/Somerville (2002)

Li Ma, Department of mathematics, Henan Normal university XinXiang, 453007, China E-mail address: lma@math.tsinghua.edu.cn

Liang Cheng, School of Mathematics and Statistics, Huazhong Normal University, WuHAN, 430079, P.R. CHINA

E-mail address: math.chengliang@gmail.com 